(RESEARCH ARTICLE)

\title{
A single centre experience of arteriovenous fistula creation for hemodialysis access in Dar es Salaam, Tanzania
}

\author{
Gabriel Francis Mtaturu 1, Obadia Venance Nyongole 2, *, Larry Onyango Akoko 2, Gudila Valentine Shirima ${ }^{3}$, \\ Jacqueline Shoo ${ }^{3}$, Victor Patrick Sensa ${ }^{1}$, Kimu Marko Njiku ${ }^{1}$ \\ ${ }^{1}$ Department of Urology, Muhimbili National Hospital, Dar es Salaam, Tanzania. \\ ${ }^{2}$ Department of Surgery, School of Medicine, Muhimbili University of Health and Allied Sciences, Dar es Salaam, Tanzania. \\ ${ }^{3}$ Nephrology unit, Muhimbili National Hospital, Dar es Salaam, Tanzania.
}

Publication history: Received on 12 October 2020; revised on 20 October 2020; accepted on 23 October 2020

Article DOI: https://doi.org/10.30574/wjarr.2020.8.1.0380

\begin{abstract}
Chronic kidney disease (CKD) is increasingly diagnosed and thus more patients are in need of hemodialysis (HD) to sustain their life. The quality of vascular access for HD should be suitable for repeated puncture and allow a fast blood flow rate for high-efficiency dialysis with minimal complications. Our study aimed to document local experience and early outcomes after arteriovenous fistula (AVF) creation for hemodialysis access including complications related to AVF creation.This was a hospital based clinical audit in which case notes of patients who had undergone AVF creation between May, 2017 and March, 2018 at Muhimbili National Hospital (MNH) were reviewed using a structured data collection tool. Information regarding preoperative assessment for AVF creation, outcome of AVF, and age of the patients were collected. Descriptive statistics were prepared and summarized as tables. A total of 57 case notes of patients who underwent AVF creation for HD access with males contributing majority of patients (77.2\%) were reviewed. The predominant age group was 41 to 60 years of age (56.2\%) with mean age 47 years and age range of 18 69 years. Three patients had procedure abandoned on the table due to sclerotic vein. The functional maturation rate was found to be $64.9 \%$ and post AVF complications in 15 patients $(26.3 \%)$. Recorded post AVF creation complications were fistula stenosis/revision (27.8\%), bleeding/haematoma (22.2\%), limb oedema (22.2\%), aneurysm (11.1\%), surgical site infection [SSI] (11.1\%) and thrombus formation (5.6\%).AVF creation for HD access is common at MNH with a functional maturation rate of $64.9 \%$, which is an acceptable rate. The preoperative vascular assessment in this survey was mainly found to be physical examination while preoperative vascular imaging was not commonly done to assess suitability of veins and arteries for AVF creation although post AVF complications are relatively few.
\end{abstract}

Keywords: Arteriovenous fistula creation; Hemodialysis

\section{Introduction}

Arteriovenous fistula (AVF) creation for hemodialysis (HD) access is the commonest indicated vascular procedure to patients with chronic kidney disease (CKD). It is well documented that vascular access is not much costly especially in relation to complications of the procedures themselves than the management of thrombotic events or infections of different types of vascular accesses [1-2]. In 2008 studies reported that 1.75 million patients worldwide received dialysis, of which 1.55 million (89\%) were on haemodialysis (HD). Nearly $62 \%$ of the HD patients were being treated in high-income countries and the remaining 38\% in low and middle-income countries [3-4].

The epidemiological pattern of diseases has changed in low income countries including Tanzania whereby there is a paradigm shift from communicable disease to Non Communicable Disease (NCD) in which both create a high demand

\footnotetext{
${ }^{*}$ Corresponding author: Dr. Obadia Nyongole

Muhimbili University of Health and Allied Sciences P. 0. Box 65001 Dar es Salaam, Tanzania.
} 
of nephrology services (dialysis and transplant). In Tanzania about 933 patients with Chronic Kidney Disease (CKD) are on dialysis and of these 256 patients are on maintenance dialysis [5-6]. The demand of AVF creation is high at Muhimbili National Hospital (MNH) with few experienced vascular surgeons of which some AVF were created by urologists with little exposure to vascular surgery [5]. So it was important to audit our practice for quality improvement, however the experience of surgeons was not part of this study. The most common reported complications include bleeding, thrombosis, infections, arterial steal syndrome, nerve injury, seroma and subclavian vein stenosis. At a later stage, there may be complications, such as surgical site infections (SSI), development of aneurysm and /or pseudo aneurysm, fistula vein stenosis, congestive cardiac failure (CCF), hand steal syndrome, ischemic neuropathy and thrombosis [7-8]. Our study aimed at documenting our local experience and early outcomes after arteriovenous fistula (AVF) creation for hemodialysis access including complications related to AVF creation.

\section{Methodology}

This was a hospital based retrospective descriptive study involving chart review of all patients who had AVF creation done at MNH, Dar es Salaam Tanzania from May 2017 to March 2018. The information of patients who had AVF created were collected by using a structured data collection tool from main operating theatre (operation log book), nephrology and urology wards and dialysis unit. Variables which were extracted from the records included; vascular image studies [CT-angiography (CT angio)], date of AVF of creation, functional fistula maturation, fistula failure, current HD access [AVF, Jugular Venous catheter (JVC) or Permanent Catheter]. The electronic data (Jeeva System) were used to ascertain if patient had vascular image done before or after fistula creation and also confirm some of the complications that occurred after surgery. The collected data was checked for completeness and entered into Statistical Package for Social Scientists (SPSS) version 20 for analysis. Categorical variables were summarized as frequency with percentages. The function and maturation of the fistula was determined by it being patent for HD access. Descriptive statistics were prepared and summarized as tables.The patient's files and all documentations regarding the management of the patients were treated with high confidentiality and respect.

\section{Results}

During the period of ten months (May, 2017 through March, 2018), fifty-seven (57) patients had undergone Arteriovenous Fistula (AVF) creation for hemodialysis (HD) access at MNH. Forty four patients (77.2\%) were males. The mean age \pm SD was $47 \pm 9$ years, median was 49 years and range of $18-69$ years with the age group of 41 to 60 years being dominant 32 patients $(56.2 \%)$.

Most of the patients 54 (94.5\%) with CKD had only vascular physical examination to assess suitability for AVF creation before and after AVF creation on top of clinical examination with minimal use of radiological imaging. Majority of patients $49(86 \%)$ had AVF as vascular access for Hemodialysis. The AVF maturation was observed in $64.9 \%$ (37 patients) of patients and 33 patients were in use of AVF for HD access during the study period. The maturity status was unknown in 14 patients (24.6\%) due to death or transfers to other centers for HD. (Table 1 )

Few patients $15(26.3 \%)$ had complications post vascular access. AVF stenosis of fistula creation (in another limb or the same limb) was the most frequently encountered complication followed by bleeding at fistula site and limb oedema after surgery. Two patients were diagnosed to have aneurysm following AVF creation (one at fistula site and another at HD access site). $\{$ Table 2$\}$

Table 1 Clinical characteristics of study participants $(\mathrm{N}=57)$

\begin{tabular}{|l|l|}
\hline Attribute & Number and percentage \\
\hline Age group (years) & \\
\hline $1-20$ & $5(8.8 \%)$ \\
$21-40$ & $10(17.5 \%)$ \\
$41-60$ & $32(56.2 \%)$ \\
$61-80$ & $10(17.5 \%)$ \\
\hline Vascular Evaluation before AVF & \\
\hline Physical examination & $54(94.5 \%)$ \\
\hline
\end{tabular}


World Journal of Advanced Research and Reviews, 2020, 08(01), 279-283

\begin{tabular}{|l|l|}
\hline Imaging (Doppler ultrasound/CT angiography) & $3(5.5 \%)$ \\
\hline Imaging after AVF creation & \\
\hline Yes & $5(5.5 \%)$ \\
No & $52(91.2 \%)$ \\
\hline Current HD access & \\
\hline JVC & $6(14 \%)$ \\
AVF & $37(86 \%)$ \\
Perm cat & 0 \\
\hline AVF maturity status & \\
\hline Matured (functional) & $37(64.9 \%)$ \\
Never matured(failed) & $6(10.5 \%)$ \\
Unknown AVF status & $14(24.6 \%)$ \\
\hline
\end{tabular}

Table 2 Complications post AVF creations

\begin{tabular}{|l|l|}
\hline Reported Complications post AVF creations & $\begin{array}{l}\text { Number and } \\
\text { percentage }\end{array}$ \\
\hline Yes & $\begin{array}{l}15(26.3 \%) \\
42(73.7 \%)\end{array}$ \\
\hline No & \\
\hline $\begin{array}{l}\text { Specific early complications Post AVF creation (one patient may have more than } \\
\text { complication }\end{array}$ & $4(22.2 \%)$ \\
\hline Bleeding/haematoma & $4(22.2 \%)$ \\
Limb oedema & $1(5.6 \%)$ \\
Thrombus formation & $2(11.1 \%)$ \\
Aneurysm & $5(27.8 \%)$ \\
AVF stenosis/revision & $2(11.1 \%)$ \\
\hline Surgical Site Infections & \\
\hline
\end{tabular}

\section{Discussion}

As more patients with end stage renal disease (ESRD) are diagnosed, the need of having secured a safe access is of paramount importance for maintenance of HD. Thus AVF is the preferred haemodialysis access. In Tanzania there are about 933 patients with ESRD on maintenance haemodialysis (MHD), for chronic dialysis or preparation for kidney transplantation (5). At MNH alone there are 256 patients on MHD weekly, which signifies the burden of ESRD at a single institution [5].

In our study (57) patients with ESRD had undergone AVF creation for HD access and majority of them were males (77.8\%), with overall predominant group aged 41 to 60 years (56\%) (table1). This is the age group which is more likely to have diabetes mellitus (DM), hypertension, peripheral vascular diseases (PVD) and obesity due to other lifestyle risks (alcohol and smoking) [1,9]. This is similar to the finding by Mushi et al in which even cost analysis was done [2]. The dominant age group found in our study also was reported to be similar to western countries as reported by Pisoni et al [10] and this shows a shift in epidemiological pattern of diseases in Sub Saharan Africa due to surge rise of NCDs possibly due to westernization of life styles $[1,5]$. 
Preoperative duplex ultrasound mapping of veins and arteries has been widely advocated to maximize the creation of native AVF for HD [11]. This is contrary to the findings of this study in which physical examination of the native vessels was a major evaluation modality for suitability of vein by $91.2 \%$ with vascular imaging being performed only in $8.8 \%$ of patients preoperatively. Poor preoperative assessment of patients for AVF creation might influence the outcome including success rates of intraoperative identification of suitable anatomy, maturation, use of access for dialysis $[11,12]$.

Although an AVF is widely regarded as the first-choice vascular access for hemodialysis, the rates of dialysis initiation with this vascular access modality remains suboptimal in many countries across the globe $[3,10$, and 12$]$ this was in line with the findings of our study in which AVF was popular by $86 \%$. Not all AVFs created will develop or mature to be subsequently useable for dialysis. Delays to use for dialysis as a result of failed maturation or complete failure (primary failure or occlusion) have been reported to be up to 50\% [13]. The accepted rate of functional AVF maturation has been established in various studies to be over $63 \%$ [3, 8, and 13]. The current study has demonstrated functional maturation rate of $64.9 \%$ (37 out of 57 patients). These results are nearly similar to those of a study done to assess predictors of fistula maturation whereby maturation rate was recorded to be $66 \%$ [7-8, 12-13].

\section{Conclusion}

AVF creation for HD access is common at MNH with a functional maturation rate of $64.9 \%$, which is an acceptable rate. Preoperative vascular imaging is not commonly done to assess suitability of veins and arteries for AVF creation although post AVF complications are relatively few. The vascular imaging should be part of routine evaluation modality preoperatively to select suitable candidates for AVF creation and those with sclerotic/thrombosed vessels to be channeled to permanent catheter for HD access.

\section{Study Limitation}

It is difficult to generalize our findings due to the fact this was a single institution (MNH) with a homogeneous cohort of ESRD patients. Therefore, there is a need for a multi-center prospective study to influence protocol development for AVF creation.

\section{Compliance with ethical standards}

\section{Acknowledgments}

We are thankful to all staff at MNH who assisted us during preparations of this work the hospital management for funding the study.

\section{Disclosure of conflict of interest}

The authors declare no conflict of interests

\section{Statement of informed consent}

Permission to conduct the study was obtained verbally because it was a clinical audit as a requirement for Masters of Science super specialization in urology in which Ethical clearance is not a requirement from Muhimbili University of Health and Allied Sciences (MUHAS) and MNH authority. Informed consent was obtained verbally from participants. The patient's files and all documentations regarding the management of the patients were treated with high confidentiality and respect.

\section{Authors' contributions}

GFM designed the study, collected data, performed data analysis and wrote the report with a manuscript. OVN participated in the study design and manuscript preparation. GS participated in the study design. LOA participated in the study design. JS participated in the study design. AM participated in the study design.

\section{References}

[1] Mathers CD, Loncar D. Projections of global mortality and burden of disease from 2002 to 2030. PLoS Med. 2006;3(11):e442. https://doi.org/10.1371/ journal.pmed.0030442. 
[2] Mushi L, Krohn M, Flessa S. Cost of dialysis in Tanzania: evidence based from provider's perspective. Health Econ Rev 2015; 5:28.

[3] Duong CM, Olszyna DP, Nguyen PD, Mclaws ML. Challenges of hemodialysis in Vietnam: experience from the first standardized district dialysis unit in ho chi Minh City. BMC Nephrol. 2015;16:122.

[4] Karopadi AN, Mason G, Rettore E, Ronco C. Cost of peritoneal dialysis and haemodialysis across the world. Nephrol Dial Transplant. 2013;28:2553-69.

[5] Furia FF, Shoo J, Ruggajo PJ, Kilonzo K, Basu G,Yeates K,et al. Developing nephrology services in low income countries: a case of Tanzania . BMC Nephrology 2019;20:378

[6] Stanifer J, Maro V, Egger J, Karia F, Thielman N, Tumer E. The Epidemiolgy of chronic Kidney Disease in Northern Tanzania: A population Based Survey. PLoS 2015 Apr 17:10(4)

[7] Stolic R." Most Important Chronic Complications of AVF for Haemodialysis" article: Medical Principles and Practice. Med Princ Pract 2013;22:220-228.

[8] Schinstock CA, Albright RC, Williams AW, Dillon JJ, Bergstralh EJ, Jenson BM, et al. Outcomes of Arteriovenous Fistula Creation after Fistula First Initiative. CJASN; American Society of Nephrology, 2011;6(8):1996 - 2002.

[9] Naghavi M, Abajobir AA, Abbafati C. Global, regional and national age-sex specific mortality for 264 causes of death, 1980-2016: a systematic analysis for the global burden of disease study 2016. Lancet. 2017;390:1151210.

[10] Pisoni R.L., Young E.W., Dykstra D.M. Vascular access use in Europe and the United States: results from the DOPPS. Kidney Int. 2002;61(1):305-316.

[11] Khawaja A, Tullett $\mathrm{K}$, Jones $\mathrm{R}$,Inston $\mathrm{N}$ : Preoperative assessment for percutaneous and open surgical arteriovenous fistula creation in patients for haemodialysis. Clinical Kidney Journal, 2019, 1-10

[12] Yoo D.W., Yoon M., Jun H.J. Successful access rate and risk factor of vascular access surgery in arm for dialysis. Vasc. Specialist Int. 2014;30 (3):33-37.

[13] Duque J.C., Martinez L., Tabbara M. Arteriovenous fistula maturation in patients with permanent access created prior to or after hemodialysis initiation. J. Vasc. Access. 2017;18 (3):185-191. 